\title{
The Consumption of Reference Resources
}

\author{
G. A. White J. A. Vaccaro H. M. Wiseman \\ Center for Quantum Computer Technology, Center for Quantum Dynamics, School of Science, \\ Griffith University, Brisbane, 4111 Australia
}

\begin{abstract}
Under the operational restriction of the U(1)-superselection rule, states that contain coherences between eigenstates of particle number constitute a resource. Such resources can be used to facilitate operations upon systems that otherwise cannot be performed. However, the process of doing this consumes reference resources. We show this explicitly for an example of a unitary operation that is forbidden by the $\mathrm{U}(1)$-superselection rule.
\end{abstract}

Keywords: Reference Frames, Superselection rules, Quantum information

PACS: 03.67.-a, 03.67.Mn, 03.65.Ud, 03.65.Ta

\section{INTRODUCTION}

Superselection rules (SSRs) are the constraint that one cannot observe coherences between eigenstates of a particular quantity [1]. They were originally discovered by Wight, Wickman and Wigner in the context of conservation laws [2]. However, it is now customary to think of them as arising from a practical restriction that one may need to consider due to the presence of a conservation law, or otherwise [1]. A SSR can in general be alleviated by an ancillary system that acts as a reference for the coherences [3]. Under a SSR there exists a group $\{T(g): g \in G\}$ of unitary operators whose effect on the system is undetectable. The notation we adopt here is that $\{T(g): g \in G\}$ is a representation of the abstract symmetry group $G=\{g\}$. Consider a system which has been prepared in the state $\rho$. As $\rho$ is indistinguishable from $T(g) \rho T^{\dagger}(g)$ for any $g \in G$, the effective state system of the system is given by the "twirl" $\mathscr{G}_{G}[\rho]$ of $\rho$ which is define as [3]

$$
\mathscr{G}_{G}[\rho]=\frac{1}{|G|} \sum_{g \in G} T(g) \rho T^{\dagger}(g)
$$

where $|G|$ is the order of $G$. This indistinguishability implies that the SSR restricts the allowed operations $\mathscr{O}$, including measurements and unitaries, that can be performed upon the system. The allowed operations are those that are $G$ invariant in the sense that [4]

$$
\mathscr{O}\left[\hat{T}(g) \rho \hat{T}^{\dagger}(g)\right]=\hat{T}(g) \mathscr{O}[\rho] \hat{T}^{\dagger}(g) \quad \forall g \in G .
$$

The definitio of the twirl in Eq. (1) is easily extended to composite systems. We restrict ourselves to composite systems where the component subsystems have the same symmetry described by the symmetry group, $G$. This means that the representation of the group $G$ for the composite system is generated by the tensor product of the representations for the subsystems. Under this restriction the twirl of a composite system in state $\rho$ is given by Eq. (1) where the composite-system operators $T(g)$ UTING (QCMC): Ninth International Conference on QCMC AIP Conf. Proc. 1110, 37 (2009) http://link.aip.org/link/?APCPCS/1110/79/1 
decomposition of the form

$$
T(g)=T_{1}(g) \otimes T_{2}(g) \otimes \cdots \quad \forall g \in G .
$$

Here $T_{i}(g)$ acts on the Hilbert space of subsystem labeled $i$.

\section{EXPLICIT EXAMPLE OF A NON U(1)-INVARIANT UNITARY}

As shown in the previous section, the effect of a SSR can be to reduce the purity of a particular state. Consider an arbitrary state, $\rho$, that may be mixed and has a vonNeumann entropy equal to $S(\rho)$. The von Neumann entropy of the effective state $\mathscr{G} \rho$ is greater that that of $\rho$, in general $[4,5]$. The increase in the von Neumann entropy is a measure of the asymmetry, $A_{G}(\rho)$, of the state $\rho$ with respect to the symmetry group $G$. The asymmetry $A_{G}(\rho)$ of $\rho$ is define as [6]

$$
A_{G}(\rho)=S\left(\mathscr{G}_{G}[\rho]\right)-S(\rho)
$$

where $S(\rho)$ is the von Neumann entropy of $\rho . A_{G}(\rho)$ has been shown to quantify the ability of an ancilla in state $\rho$ to act as a reference and partially alleviate the effects of the $G$ SSR [6].

In general the use of a reference ancilla to perform a non U(1)-invariant operation on the system may increase the asymmetry of the system with respect to the U(1) group of phase translations. Using a reference ancilla repeatedly in this way will reduce the reference ability of the ancilla. This effect has been studied explicitly for the case where a reference is used to facilitate a phase measurement [7]. Here we wish to examine this process for the case of a non U(1)-invariant unitary operation. Consider a system that is in the vacuum state

$$
|\psi\rangle_{S}=|0\rangle_{S}
$$

The operation $F_{\mathrm{S}}$ on the system for which

$$
F_{S}\left(|0\rangle_{S}\right)=\frac{1}{\sqrt{2}}\left(|0\rangle_{S}+e^{i \theta}|1\rangle_{S}\right)
$$

is forbidden under the SSR. However, an analogous operation can be performed on the coherent subspaces of the combined reference ancilla and system as follows. We defin an analogous operation on the basis $\left\{|n\rangle_{R}|0\rangle_{S},|n-1\rangle_{R}|1\rangle_{S}\right\}$ of the coherent subspaces representing $n$ particles by

$$
F_{\mathrm{RS}}^{(n)}|n\rangle_{R}|0\rangle_{S}=\frac{1}{\sqrt{2}}\left(|n\rangle_{R}|0\rangle_{S}+|n-1\rangle_{R}|1\rangle_{S}\right)
$$

for $n=1,2, \ldots, N$. One can perform this operation with an ancilla say, of the form $|n\rangle$ and produce the the right hand side of (7). However, upon performing the operation one can easily see that the partial trace over the reference would leave the system in a maximally mixed state. To minimize this effect use a phase state of the form [8] 


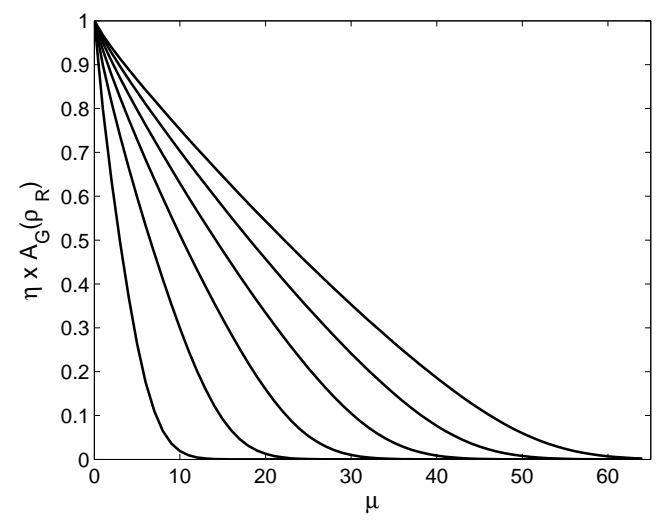

FIGURE 1. The consumption of the reference ancilla is demonstrated by the reduction of the normalized asymmetry for reference ancilla of $M=5,10,15,20,25$ and 30 as a function of the number of times, $\mu$, the reference is used. Here the normalization, $\eta$, is given by $\log _{2}\left[D_{R}\right]$.

$$
|\psi\rangle_{R}=\frac{1}{\sqrt{N+1}} \sum_{n=0}^{N} e^{i \theta n}|n\rangle_{R},
$$

as the state of the reference ancilla. This state has the property that it maximizes the asymmetry of the reference state which has at most $N$ particles. To further simplify matters we set $\theta=0$. The forbidden operation, $\mathscr{F}_{S}$. can be mapped onto a globally G-invariant operation that acts on the combined state to give

$$
\begin{aligned}
|\Psi\rangle_{R S} \equiv F_{\mathrm{RS}}|\psi\rangle_{\mathrm{R}}|0\rangle_{S} & =\frac{1}{\sqrt{2(N+1)}} \sum_{n=1}^{N}\left[|n\rangle_{R}|0\rangle_{S}\right. \\
& \left.\left.+|n-1\rangle_{R}|1\rangle_{S}\right)\right]+\frac{1}{\sqrt{N+1}}|0\rangle_{R}|0\rangle_{S}
\end{aligned}
$$

This state is entangled. Therefore the reduced state of the reference ancilla is mixed. Consider a system in the vacuum state $|0\rangle$. We begin with a reference ancilla in a phase state and perform the forbidden operation outlined above. The reduced state of the reference is then given by

$$
\rho_{R}^{\prime}=\operatorname{Tr}_{S}[|\Psi\rangle\langle\Psi|]
$$

The reference ancilla is then used to facilitate the same operation on another system which is also prepared in the state $|0\rangle$. This process is repeated $\mu$ times and the resources, i.e. the asymmetry, of the reference ancilla is consumed as $\mu$ increases. The consumption of the reference ancilla for reference ancillae of various size is shown in figur 1 as a function of the number of times, $\mu$, the reference is used to perform the U(1)-SSR forbidden operation, $F_{S}$.

Consider now the reduced state of the system. Due to the entanglement between the system and the reference ancilla, the reduced state of the system is mixed and therefore the fidelit between the system with the state on the right hand side of (6) is not perfect. 


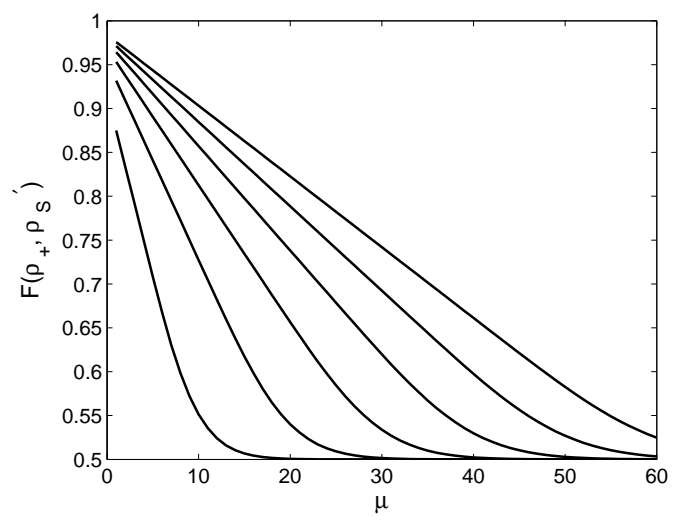

FIGURE 2. The degradation in fidelit of the system state with a $|+\rangle$ state for $M=5,10,15,20,25$ and 30 where $\mu$ is the number of times the reference has been used.

To see this we firs defin the fidelit of the operation as

$$
F\left(\rho_{S}^{\prime}, \rho_{+}\right)=\operatorname{Tr}\left[\rho_{S}^{\prime}|+\rangle\langle+|\right]
$$

where

$$
\rho_{S}^{\prime}=\operatorname{Tr}_{R}\left[F_{S} \rho_{R}|0\rangle_{S}\langle 0|\right] \text { and }|+\rangle=\frac{1}{\sqrt{2}}(|0\rangle+|1\rangle) .
$$

The fidelit the fina state of the system shown in Figure 2. We see that the reference's ability to facilitate a non U(1)-invariant unitary reduces as the reference resources of the reference ancilla are consumed. The similarity of the trends in figure 1-2 show that as the reference show that as the reference is consumed its ability to facilitate non U(1)invariant operations diminishes.

\section{ACKNOWLEDGMENTS}

This work was supported by the Australian Research Council

\section{REFERENCES}

1. S. D. Bartlett, T. Rudolph, and R. W. Spekkens 79, 555 (2007).

2. G. C. Wick, A. S. Wightman, and E. P. Wigner 88, 102 (1952).

3. Y. Aharanov, and L. Susskind 155, 1428 (1967).

4. S. D. Bartlett, and H. M. Wiseman 91, 097903 (2003).

5. H. M. Wiseman, and J. A. Vaccaro 91, 097902 (2003).

6. J. A. Vaccaro, F. Anselmi, H. M. Wiseman, and K. Jacobs, Phys. Rev. A 77, 032114 (2008).

7. S. D. Bartlett, T. Rudolph, R. W. Spekkens, and P. S. Turner., New. J. Phys. 8, 58 (2006).

8. D. T. Pegg, and S. M. Barnett, Europhys. Lett. 6, 483 (1988). 